\title{
DALLE ESIGENZE ENERGETICHE SULLA TERRA ALL'ESPLORAZIONE DI MARTE PASSANDO PER I COLORI DELLA CAPPELLA SISTINA: PRESENTE E FUTURO DEL FOTOVOLTAICO E DEI COMBUSTIBILI SOLARI
}

\author{
ALESSANDRO ABBOTTO (*) \\ Nota presentata dal m.e. Stefano Maiorana \\ (Adunanza del 16 novembre 2017)
}

SuNTO. - La sfida delle energie rinnovabili è giunta oggi ad un passaggio strategico. Mentre è vero che l' $80 \%$ dell'energia proviene ancora oggi dai combustibili fossili, il ruolo delle rinnovabili è in inarrestabile crescita. Tuttavia, non si tratta solo di una transizione tecnologica ma, da un lato, è la risposta necessaria alle esigenze del pianeta, quali la crescita demografica, le emergenze climatiche, la crescita dei paesi più poveri e, dall'altro, è un presupposto a prospettive di medio-lungo termine come la colonizzazione di Marte. I ricercatori sono quindi impegnati a sviluppare soluzioni innovative, pulite ed efficienti per conseguire tali obiettivi. In particolare i coloranti organici, sostanze organiche come i farmaci o i cristalli liquidi appositamente progettate, rappresentano una delle frontiere più affascinanti della chimica per produrre energia rinnovabile a basso costo e in modo pulito. Come i veri colori degli affreschi della Cappella Sistina, voluti da Michelangelo, sono ora emersi e visibili a tutti, anche in questo campo solo alcuni coloranti e non altri sono in grado di rispondere con efficacia alla sfida energetica. Lo scienziato, in particolare il chimico, con conoscenza dello stato dell'arte e fantasia, è impegnato nella progettazione di nuove molecole dotate dei colori opportuni.

$* * *$

ABSTRACT. - The challenge of renewable energies has now come to a strategic step. While

(*) Centro di Ricerca sull'Energia Solare, Dipartimento di Scienza dei Materiali, Università di Milano-Bicocca, Milano, Italy. E-mail: alessandro.abbotto@unimib.it 
$80 \%$ of the energy still comes from fossil fuels, the role of renewable energies is in constant growth. The challenge of renewables is not only a technological transition, but also the indispensable response to the needs of the planet, from the incessant demographic growth to climatic emergencies and to the development of the poorest countries, and eventually to medium to long term challenges such as the colonization of Mars. Researchers are therefore committed to developing innovative, clean and efficient solutions to meet such challenge. In particular, organic dyes, organic molecules such as those founds in drugs or liquid crystals, represent key actors of one of the most fascinating chemistry frontiers to produce low cost and clean renewable energy. As the true colors of the Sistine Chapel frescoes, wanted by Michelangelo, have now emerged and visible to everyone, also in this field only specific colored dyes are able to address effectively the energy challenge. The scientist, especially the chemist, starting from the state of the art and innovation, is engaged in the design of new molecules owning the correct colors.

\section{INTRODUZIONE}

Il dilemma energetico ha raggiunto oggi una fase critica. Importanti emergenze planetarie richiedono soluzioni energetiche efficaci ed immediate. L'esplosione della popolazione nell'ultimo secolo, l'emergenza climatica e l'inquinamento ambientale non consentono più di mantenere le strategie energetiche finora utilizzate. Nel 2014 circa 1.2 miliardi di persone (poco meno del 20\% della popolazione mondiale) non hanno ancora accesso all'elettricità.[1] In particolare, in molte regioni centrali dell'Africa, nella zona sub-sahariana e nella regione oceanica, l'accesso alla corrente elettrica copre solo il 10-40\% della popolazione, rendendo impossibili importanti operazioni quotidiane come la conservazione di farmaci sensibili al calore, vaccini e antibiotici o il semplice utilizzo di cibi deperibili. Le politiche energetiche del nuovo secolo sono quindi urgentemente chiamate a considerare e risolvere queste criticità. Una politica energetica sostenibile deve innanzitutto affrontare la povertà nel mondo, rendendo accessibile a tutta la popolazione mondiale energia elettrica, cibo e acqua. Dall'altra parte l'emergenza energetica si origina anche dall'inevitabile diminuzione, finanche esaurimento, delle fonti fossili di energia, intrinsecamente non rinnovabili se non nella scala dei milioni di anni. Secondo l'associazione scientifica ASPO (Association for the Study of Peak Oil) la produzione petrolifera mondiale ha già raggiunto o raggiungerà il suo picco nell'attuale decennio. Da questo momento quindi le disponibilità energetiche tradizionali continueranno a diminuire, rendendo impossibile il mantenimento delle attuali tecnologie. L'effetto della crescente scarsezza delle 
fonti fossili non solo avrà inevitabili ripercussioni economico-finanziarie (già oggi le nuove tecnologie di reperimento delle fonti fossili, quali il shale oil/gas, le perforazioni artiche o oceaniche, le sabbie bituminose, consentono di accedere alle fonti fossili solo a costi molto superiori a quelli tradizionali) ma impedirà, tra l'altro, di accedere alla materia prima di importanti intermedi alla base dell'industria chimica.[2]

La sfida delle energie rinnovabili è giunta oggi ad un passaggio strategico. Mentre è vero che l'80\% dell'energia proviene ancora oggi dai combustibili fossili, il ruolo delle rinnovabili è in inarrestabile crescita. Già in questi anni, sia in Italia che in altri paesi, nelle giornate soleggiate estive il 100\% dell'energia elettrica è prodotta esclusivamente da fonti rinnovabili, un fatto noto a pochi.[3] Come detto, la sfida delle rinnovabili non è solo una transizione tecnologica, ma la risposta necessaria alle esigenze del pianeta, dalla crescita demografica alle emergenze climatiche e alla crescita dei paesi più poveri, fino ad affrontare sfide di medio-lungo termine come la colonizzazione di Marte. I ricercatori sono quindi impegnati per sviluppare soluzioni innovative, pulite ed efficienti per rispondere alla sfida.

La più importante fonte di energia oggi disponibile alla specie umana è il Sole. Il Sole invia oggi sulla terra una quantità di energia almeno 5000 volte superiore a quella che viene utilizzata. In termini di potenza energetica il consumo di energia mondiale è di circa $18 \mathrm{TW}$ ed è previsto aumentare fino a oltre $30 \mathrm{TW}$ entro il 2015. Per contro l'energia che ci proviene dal Sole risulta poco meno di 100,000 TW. L'energia solare, insieme all'energia geotermica e a quella gravitazionale (maree) costituiscono le tipologie di energia rinnovabili. In particolare possiamo classificare, in base alla tipologia di energia prodotta, l'energia solare in tre grandi categorie: 1) energia termica (riscaldamento, combustibili); 2) energia elettrica (fotovoltaico); 3 ) reagenti e combustibili per produzione e trasporto (fotosintesi naturale e artificiale) (Fig. 1). [4]

Nel campo dell'energia solare il ruolo delle molecole chimiche, in particolare delle molecole organiche, è in continua crescita. In particolare, la classe molecolare dei coloranti organici, sostanze organiche come i farmaci o i cristalli liquidi appositamente progettate, rappresenta una delle frontiere più affascinanti della chimica per produrre energia rinnovabile a basso costo e in modo pulito. Ad ogni molecola potenzialmente utile per catturare l'energia solare è associata una precisa struttura chimi$\mathrm{ca}$, appositamente ingegnerizzata. Solo certe componenti molecolari e non altre, potranno conferire alla molecola le giuste proprietà per fungere 
da componenti efficienti e stabili in dispositivi solari. In altri termini, poiché ad ogni molecola in grado di interagire efficacemente con la radiazione solare, è associata una precisa banda di assorbimento della luce solare, in particolare la porzione visibile, possiamo dire che un preciso colore, $\mathrm{e}$ non un altro, conferirà alla molecola le caratteristiche desiderate. Dopo secoli in cui i falsi colori della Cappella Sistina, a causa del fumo delle candele e dell'inquinamento ambientale, sono quelli che tutto il mondo ha visto, oggi un accurato procedimento di restauro e impianto di illuminazione (che ha riprodotto la naturale illuminazione ai tempi michelangioleschi), ha restituito i veri colori degli affreschi, quelli che Michelangelo ha progettato e dipinto. Come i veri colori degli affreschi della Cappella Sistina sono ora emersi e visibili a tutti, anche nel campo dell'energia solare solo alcuni "colori" e non altri sono in grado di rispondere con efficacia alla sfida energetica. Lo scienziato, in particolare il chimico, con la conoscenza dello stato dell'arte e molta fantasia, è impegnato nella progettazione di nuove molecole dotate dei "colori" opportuni.

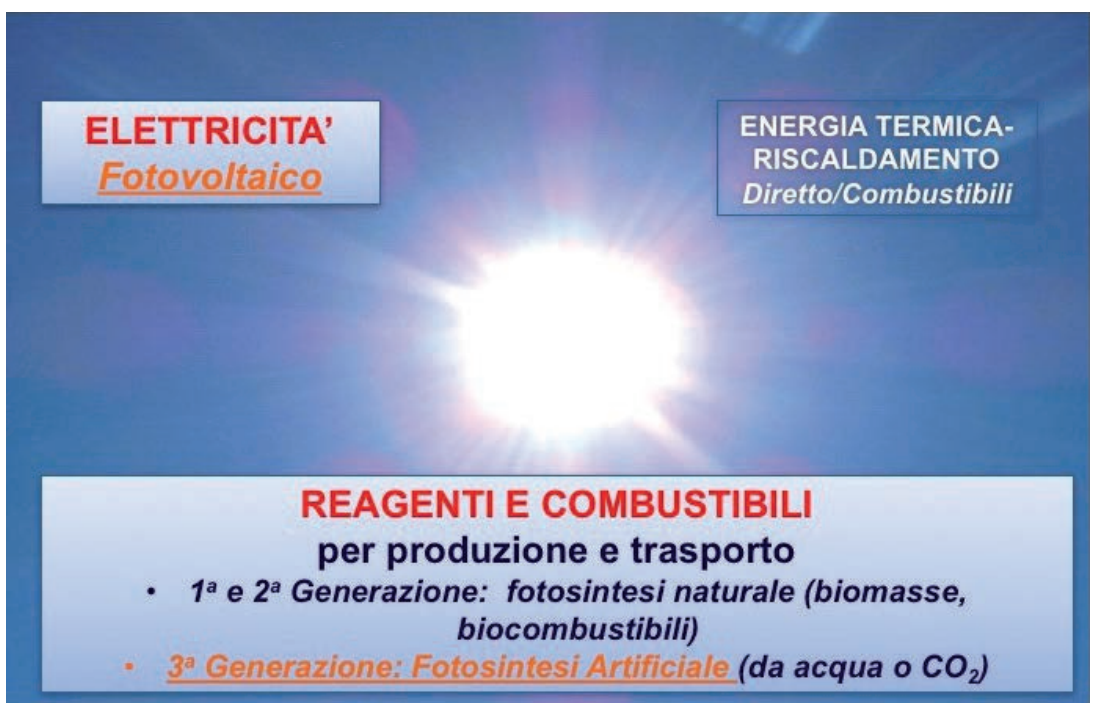

Fig. 1.

L'utilizzo delle proprietà molecolari porta a ricadute non solo in ambito strettamente accademico ma consente di produrre dispositivi a costo minore e in modo più facile, di disporre di un'ampia varietà di caratteristiche estetiche ed architettoniche (si pensi ad esempio alla 
varietà dei colori disponibili per un pannello fotovoltaico), di rendere possibile l'integrazione immediata con dispostivi, edifici, automobili e altri oggetti tecnologici, nonché materiali di qualsiasi genere, dalla plastica ai metalli e finanche tessuti e vestiti, ed infine di permettere di ispirarsi alla Natura (si pensi ad esempio, per quanto riguarda l'energia solare, alla fotosintesi clorofilliana).

Ingegnerizzare una molecola per dispositivi solari significa pertanto progettare accuratamente tutte le componenti chimiche della struttura, non solo per conferire le giuste caratteristiche intrinseche, ad esempio la capacità di assorbire efficacemente la luce solare e di convertirla, ma anche per dialogare e interfacciarsi opportunamente con materiali di altro tipo (ad esempio nanoparticelle di ossidi o contatti metallici) e altre componenti presenti nel dispositivo (Fig. 2).

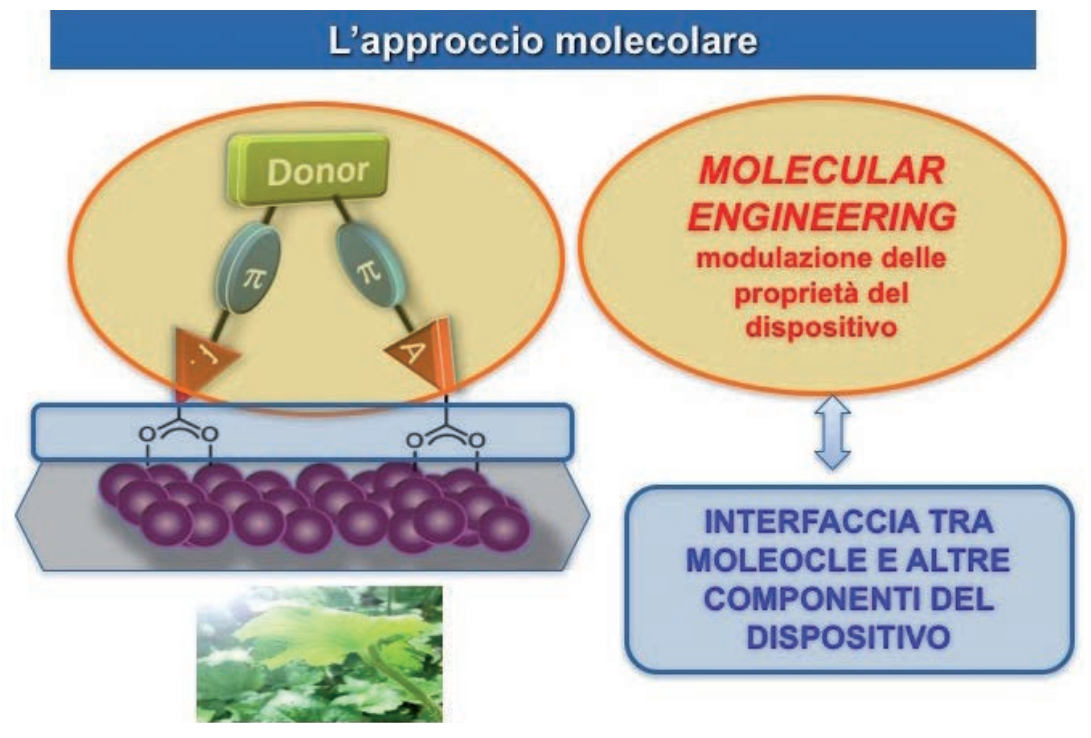

Fig. 2.

\section{Molecole E Fotovoltaico Di Ultima Generazione}

La Fig. 3, presa dal National Renewable Energy Laboratory statunitense,[5] riassume graficamente l'attuale stato della tecnologia fotovoltaica. Se ci si limita alle cosiddette celle singole allora è possibile classifi- 
care la tecnologia fotovoltaica in tre generazioni: 1) prima generazione, a base di silicio; 2) seconda generazione, a base di film sottili; 3 ) terza generazione, a base di molecole organiche o ibride. Questa sezione tratterà della terza generazione, con particolare riferimento all'utilizzo dei coloranti organici. Per quanto riguarda questa tipologia troviamo essenzialmente tre tipi di dispositivi: 1) dispostivi a colorante molecolare (dye-sensitized solar cells, DSSC); 2) dispositivi completamente organici (organic photovoltaics, OPV); 3 ) celle solari a base di perovskiti ibride organicheinorganiche (perovskite solar cells, PSC). Di queste le più promettenti sono la prima e la terza, con efficienze di conversione energetica, ovvero il rapporto tra l'elettricità prodotta (elettroni) e la luce assorbita (fotoni), ormai superiore al $20 \%$ e vicina al record delle celle a silicio del $26 \%$. Si noti che questi valori si riferiscono alla scala di laboratorio, mentre per la scala industriale le efficienze sono leggermente inferiori.

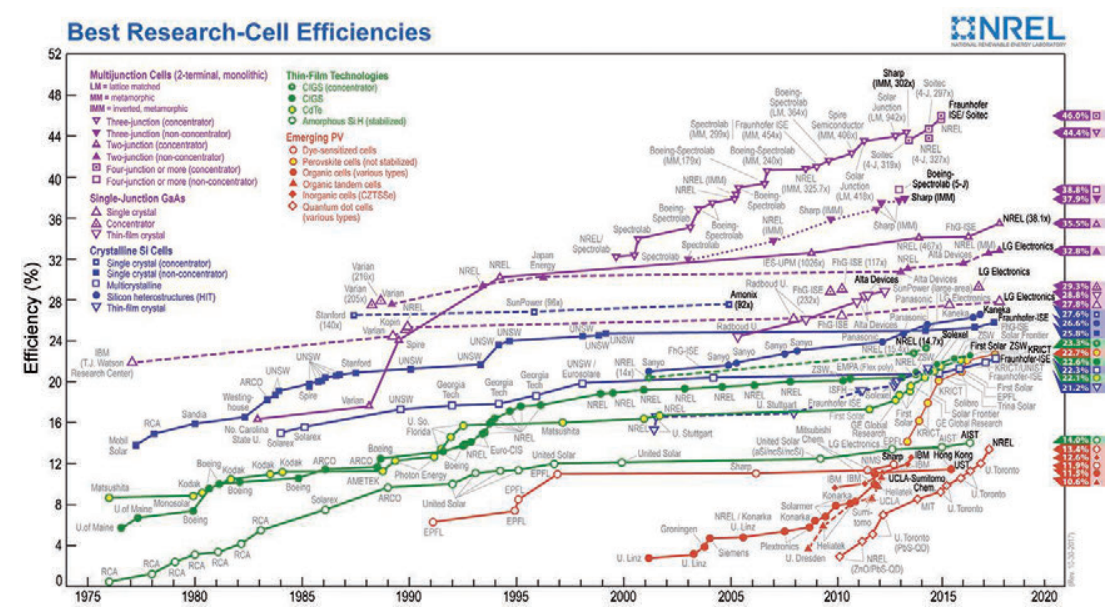

Fig. 3.

A fronte di efficienze leggermente inferiori, le celle di terza generazioni hanno tuttavia caratteristiche peculiari che le rendono uniche e alternative alle celle a silicio. Ad esempio possono dar luogo a dispositivi trasparenti e coloranti, per integrazioni architettonica (buildingintegration) al posto delle vetrate convenzionali, flessibili, leggeri e facilmente integrabili in altri dispostivi elettronici. Tra le caratteristiche più interessanti troviamo la possibilità di lavorare in luce diffusa (alba, crepuscolo, interni di edifici), dove il silicio non funziona, e di poter 
essere prodotti a bassissimo costo. Si pensi infatti le nuove celle molecolari utilizzano circa 300 volte meno materiale di una cella a silicio, materiale inoltre che non deve avere gli eccezionali requisiti di purezza $(99.99999 \%)$ del silicio solare. Di fatto l'industria chimica che produce cristalli liquidi per display e televisori e farmaci è già in grado di produrre i coloranti per fotovoltaico in modo economico ed efficiente.

Pur essendo stata introdotta solo da pochi anni questa tecnologia sta già arrivando ai primi esempi di installazioni esterne. Alcuni esempi sono riportati in Fig. 4 tra questi evidenziamo le due installazioni a Milano, tra le prime al mondo, in occasione dell'EXPO 2015 (padiglione austriaco) e nella nuova piazza Gae Aulenti.
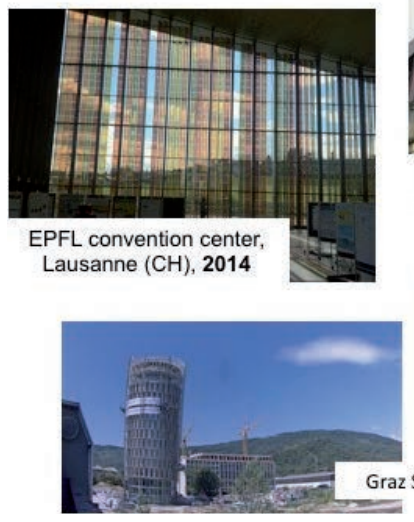

Negli ultimi anni il centro MIB-SOLAR si è occupato soprattutto di celle DSSC, in particolare dello sviluppo di nuovi coloranti organici che fungono da fotosensibilizzatori, ovvero le molecole deputate a raccogliere la luce solare e convertirla in un flusso di elettroni. I fotosensibilizzatori sono pertanto le componenti più importanti di una cella DSSC, la cui struttura è graficamente riportata in Fig. 5. Più che entrare nel dettaglio del funzionamento di una cella DSSC è qui importante descrivere la struttura molecolare del fotosensibilizzatore, schematizzata in Fig. 6. Il fotosensibilizzatore in Fig. 6 è una molecola organica comprendente sistemi aromatici (derivati del benzene) e doppi e tripli legami, che conferiscono la peculiare proprietà di essere colorate e 
assorbire la porzione visibile della luce solare in modo efficiente. Tuttavia questo non è sufficiente. Per poter trasformare efficacemente fotoni in elettroni la molecola deve essere opportunamente ingegnerizzata comprendendo al suo interno sotto-unità molecolari dotate di precise proprietà elettroniche (caratteristiche di ricchezza o deficienza elettronica, doppi e tripli legami adiacenti). Il chimico progetta e costruisce molecole di questo tipo proprio sviluppando nuovi frammenti molecolari elettron-ricchi o elettron-poveri e combinandoli in una struttura molecolare come quella riportata in Fig. 6. Ogni combinazione di frammenti molecolari porta a molecole diverse dotate, ciascuna, di un particolare "colore". E' questa caratteristica peculiare che farà si che alcune molecole funzioneranno meglio di altre.

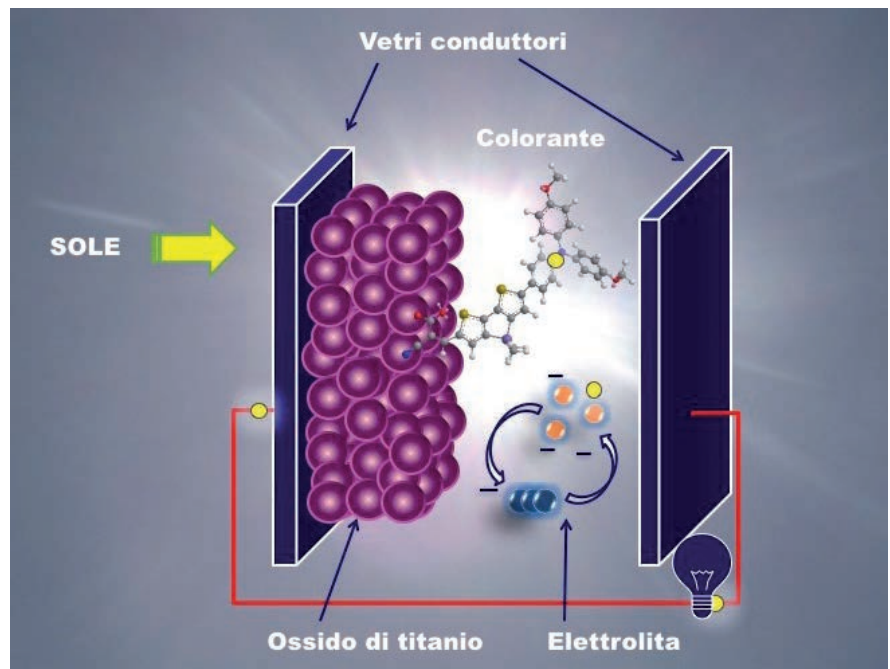

Fig. 5

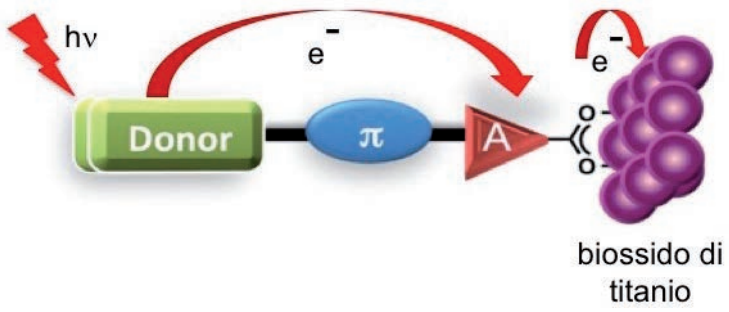

Fig. 6. 
Recentemente il nostro gruppo di ricerca ha introdotto un nuovo tipo di architettura molecolare che oggi è ampiamente usato dalla comunità scientifica (Fig. 7).[6] Questo esempio ben rappresenta come una opportuna, ma allo stesso tempo semplice, progettazione molecolare possa portare benefici in termini di proprietà molecolari chiave e prestazioni finali del dispositivo. Nella parte in alto è rappresentata l'architettura convenzionale, riportata in letteratura. In questa architettura i frammenti molecolari sono disposti approssimativamente linearmente. Nella nuova architettura, da noi proposta e chiamata multi-ramificata, riportata nella parte inferiore della Fig. 7, il percorso degli elettroni lineare è sostituito da 2 o più percorsi molecolari, per favorire la trasformazione da fotoni ad elettroni e il trasferimento degli elettroni agli elettrodi e quindi al circuito elettrico esterno. Inoltre la struttura ramificata permette di migliorare le proprietà di assorbimento della luce e la stabilità dei legami (due al posto di uno) verso le nanoparticelle di biossido di titanio, rappresentate in viola, a loro volta presenti in forma di film sull'elettrodo. Nel complesso pertanto la nuova architettura ha permesso di ottenere sistemi molecolari e dispositivi con migliori proprietà di assorbimento della luce, trasporto di elettroni e stabilità. Alcuni composti di questa serie sono stati oggetto di alcuni brevetti internazionali depositati insieme a ENI SpA.[7]

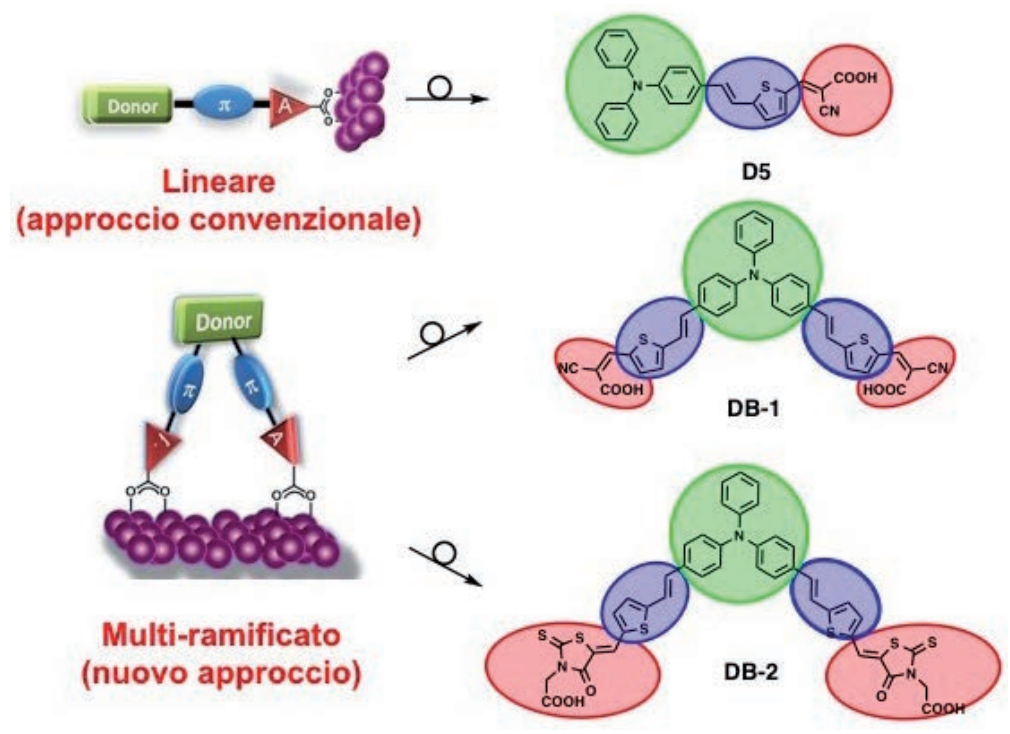

Fig. 7. 
In un altro esempio più recente, del 2017, abbiamo invece legato chimicamente una molecola di colorante al grafene, al fine di sfruttare le importanti proprietà di quest'ultimo in ambito fotovoltaico (Fig. 8).[8] Questo risultato, ottenuto insieme all'Università di Padova, pioniere della chimica del nanocarbonio (fullereni, nanotubi di carbonio e grafene) è il primo esempio al mondo di diade colorante-grafene per fotovoltaico di nuova generazione. Pur non raggiungendo efficienze elevate abbiamo verificato come la diade colorante-grafene sia più efficiente della molecola isolata. In entrambe i casi descritti, si è posto particolare attenzione alla stabilità, oltre che all'efficienza. Infatti solo un sistema molecolare che sia stabile per molti anni potrà consentire la commercializzazione dei dispositivi corrispondenti. La stabilità rappresenta pertanto un requisito essenziale che deve sempre accompagnare le altre proprietà molecolari anche se nella maggior parte dei report in letteratura questo aspetto viene, erroneamente, trascurato.

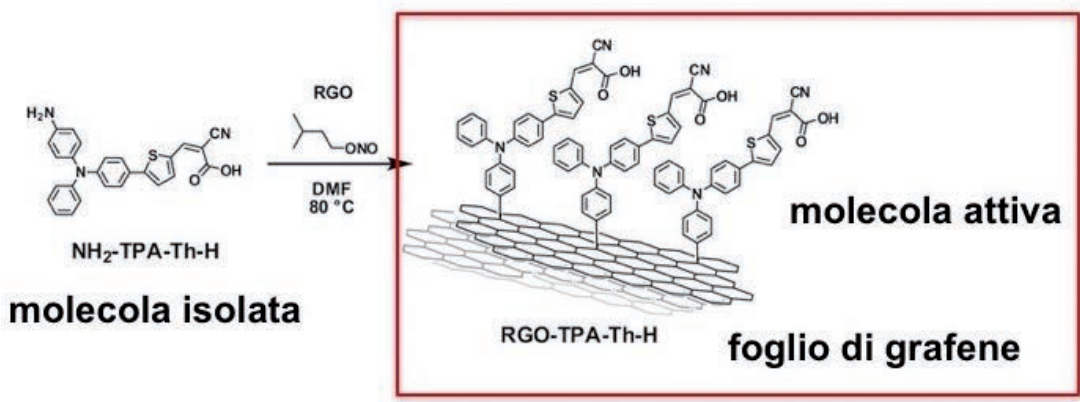

Fig. 8.

Nell'ultimo esempio che vi presentiamo, anch'esso del 2017, abbiamo affrontato un altro aspetto sempre più rilevante nell'industria chimica e tecnologica in generale, ovvero l'utilizzo di metodi di produzione completamente eco-sostenibili. In particolare, abbiamo affrontato il problema legato all'utilizzo di solventi organici volatili e tossici sia nella preparazione sia nella stessa costituzione del dispositivo (nel caso specifico l'acetonitrile). Sarebbe infatti un paradosso proporre nuovi dispositivi solari e sostenibili ma allo stesso tempo utilizzare composti tossici per la loro produzione. Sfruttando una chimica molto recente, abbiamo sostituito il solvente organico tossico con una miscela chiamata Deep Eutectic Solvent a base di colina (usato come mangime per i 
polli) e la glicerina (o glicerolo), ampiamente usata in preparazione medicinali, cosmetiche e come additivo alimentare (E422) e facilmente ottenibile per saponificazione di grassi e olii (Fig. 9).[9] Entrambi i composti non solo non sono tossici ma sono ampiamente disponibili a costi molti bassi. Nella copertina che la rivista ci ha attribuito abbiamo intitolato "drinkable solvents", per sottolineare appunto l'aspetto di eco-sostenibilità. Le efficienze energetiche sono risultate minori che nel caso dell'utilizzo del solvente tradizionale ma, riteniamo, ampiamente compensate dall'elevata sostenibilità del prodotto, un requisito chiave per la sua ampia commercializzazione.

ELETTROLITA

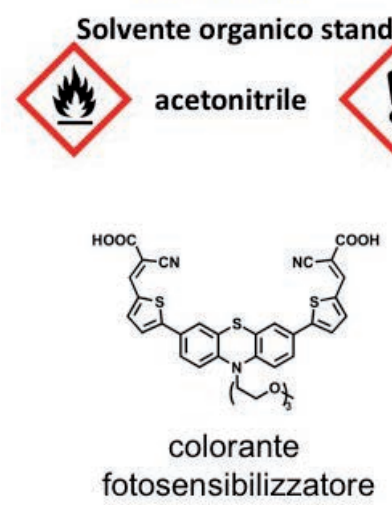

DES

Cloruro di colina : Glicerina 2:1 $40 \%$ acqua

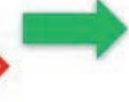

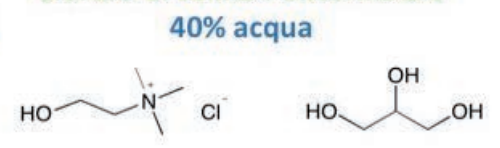

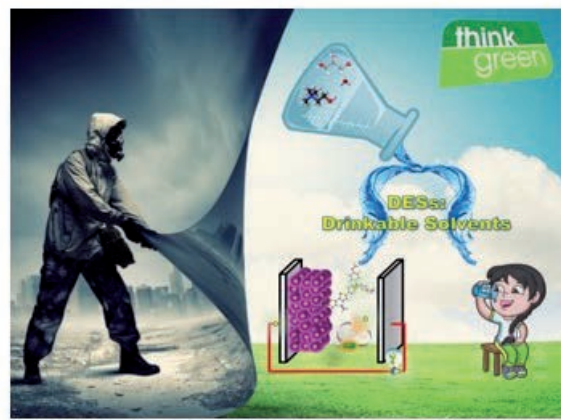

Fig. 9.

\section{MoleCole E COMbUSTIBILI SOlARI DI ULTIMA GENERAZIONE}

Nel capitolo 2 abbiamo affrontato la produzione solare di elettricità. Tuttavia, seppur auspicabile,[4] la completa conversione verso l'elettricità di tutte le necessità energetiche è al momento non ottenibile allo stato delle conoscenze attuali. Di fatto oggi il $60 \%$ dell'energia prodotta non è legato all'elettricità ma ai combustibili, in particolare per trasporto (auto, navi ed aerei) e per la sintesi di intermedi chimici. Per quanto riguarda l'autotrasporto in questi mesi stiamo assistendo ad una rivolu- 
zione. Dal 2016 in Norvegia, pur essendo il principale produttore europeo di petrolio, il 30\% del parco automobilistico è elettrico o ibrido. Purtroppo l'Italia non rientra nella classifica dei primi dieci paesi col maggior tasso di trasporto elettrico. Importanti aziende automobilistiche (Volvo, Mercedes, Volkswagen) hanno annunciato l'abbandono completo di automobili a trazione fossile (benzina, diesel) oppure il completamento di tutta la gamma, compresi i brand affiliati, con modelli di autovetture elettriche. Ormai la transizione è segnata. Tuttavia la trazione elettrica non è la soluzione di tutti i problemi. A parte gli attuali alti costi delle vetture elettriche, l'autonomia è limitata a meno, in alcuni casi molto meno, di $500 \mathrm{~km}$ e i tempi di ricarica possono essere di alcune ore (alcune decine di minuti nei casi più tecnologicamente avanzati). Inoltre, pur esistendo prototipi di navi fotovoltaiche che hanno circumnavigato il globo (MS Tûranor PlanetSolar) e di aerei fotovoltaici che hanno coperto distanze ragguardevoli (Solar Impulse 2, percorso Giappone-Hawaii, circa $8000 \mathrm{~km}$ ), non è pensabile né a breve né a medio-lungo termine, di disporre di navi e aerei commerciali a trazione elettrica (Fig. 10). I combustibili per trasporto rappresentano pertanto l'unica soluzione tecnologica per le prossime decine di anni.

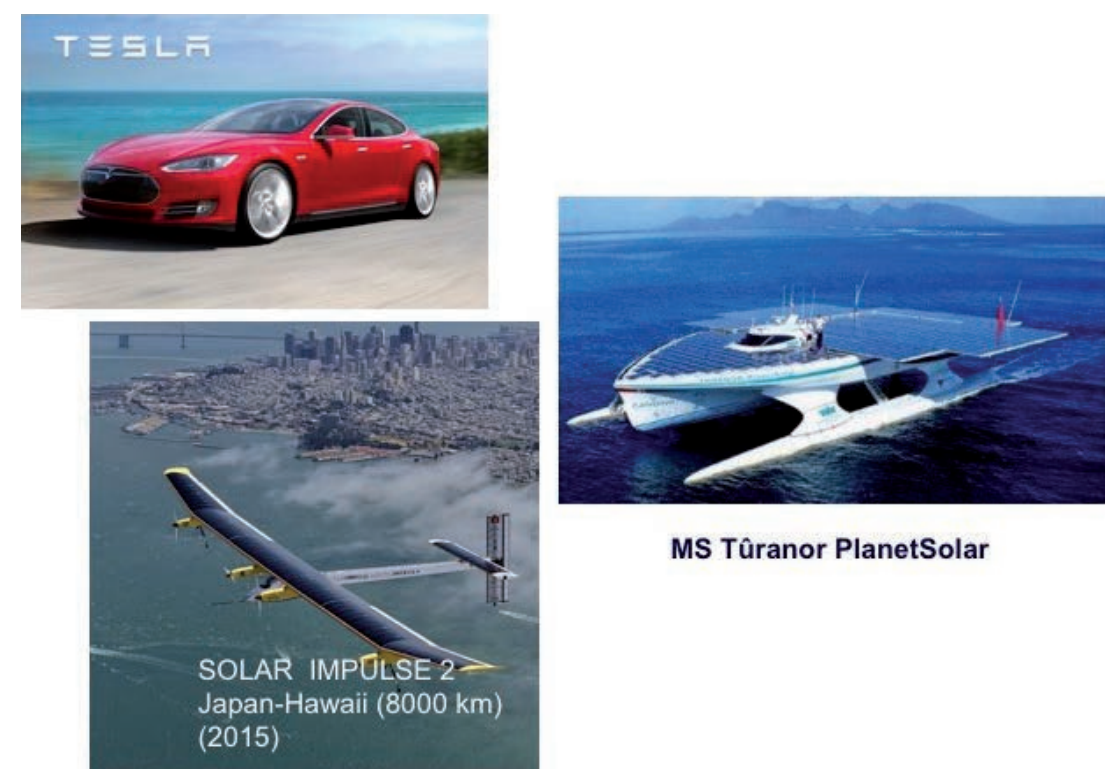

Fig. 10. 
Se non consideriamo le biomasse, l'unico combustile per trasporto sostenibile è l'idrogeno.[1] L'idrogeno è il combustibile ideale perché ha carbon footoprint nulla e per combustione produce solo vapore acqueo ed elettricità, quest'ultima nei motori a celle a combustibile. I primi esempi di utilizzo di serie dell'idrogeno per trasporto sono apparsi sul mercato proprio quest'anno (Fig. 11).

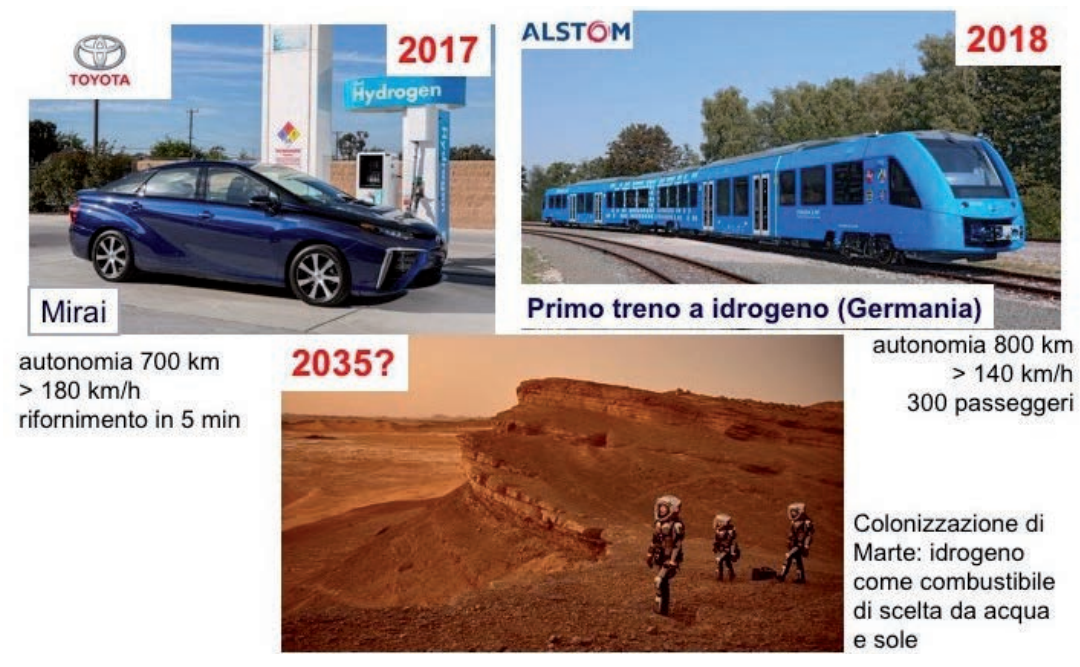

Fig. 11.

La Toyota ha commercializzato la prima autovettura di serie a idrogeno (Toyota Mirai) e nel 2018 in Germania prenderà servizio il primo treno al mondo a trazione a idrogeno. In entrambi i casi le prestazioni e l'autonomia sono comparabili, se non superiori, a quelle dei modelli a trazione convenzionale. Nel caso del modello automobilistico il completo rifornimento viene effettuato in circa 3 minuti, ovvero in tempi analoghi a quelli convenzionali. L'idrogeno è anche il combustibile di scelta, se non l'unico possibile, per la colonizzazione di Marte, dove il trasferimento dalla Terra di combustibili fossili è impensabile e lo sfruttamento dell'energia fotovoltaica è reso difficile dalla maggiore distanza dal Sole e dalle continue tempeste di sabbia. Esiste anche un ambizioso piano nazionale per la mobilità a idrogeno che prevede 27,000 veicoli a idrogeno per il 2025, 8 milioni (20\% del totale) di veicoli per il 2050, 23,000 autobus e 20 stazioni di rifornimento per il 2020, che diventeranno 5,000 entro il 2050. Due anni fa è stata inaugurata nell'autostrada del Brennero 
a Bolzano Sud la prima stazione di rifornimento di idrogeno (Fig. 12). Non mancano, infine, applicazioni per trasporti di dimensioni maggiori, come il trasporto su mare. Lo scorso luglio è partito da Parigi il giro del mondo di "Energy Observer", la prima nave ad idrogeno, che prende il combustibile direttamente dall'acqua su cui naviga dopo dissalazione e trasformazione in idrogeno con un processo di elettrolisi.

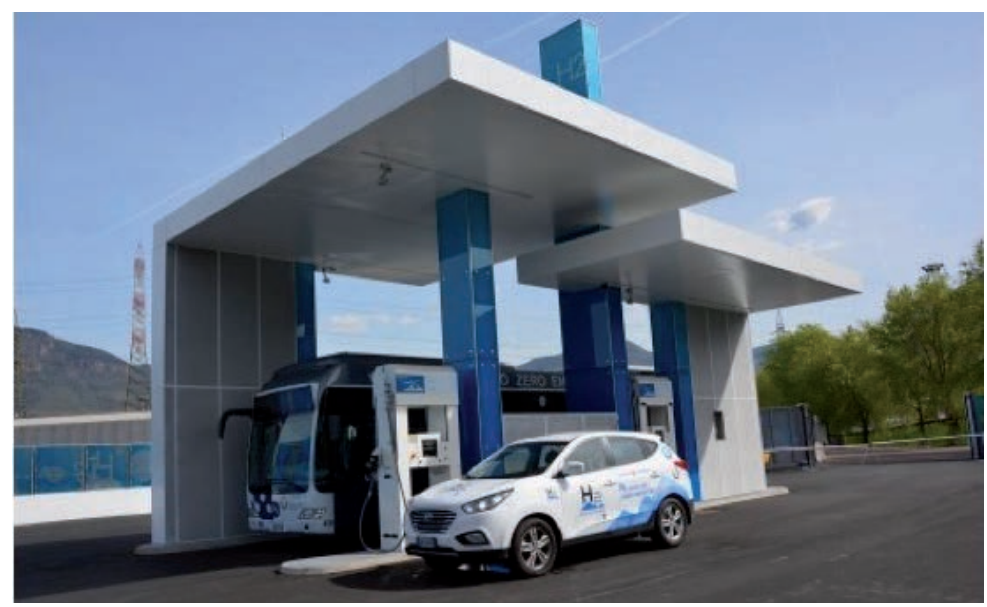

Fig. 12.

Tuttavia l'idrogeno non è una fonte di energia, non essendo presente in modo naturale sulla Terra, bensì un vettore energetico. Deve essere quindi ottenuto da altre fonti. Attualmente il metodo principale di produzione dell'idrogeno è lo steam reforming da metano, un combustibile fossile. Quindi l'uso dell'idrogeno è pulito ma non lo è la sua produzione. Il vantaggio netto in termini di eco-sostenibilità è pertanto nullo. Per accedere ad un'economia dell'idrogeno veramente sostenibile è necessario quindi produrre idrogeno in altro modo. Negli ultimi anni la ricerca sta concentrando gli sforzi nella produzione di idrogeno a partire da acqua e luce solare, in un processo, chiamato fotosintesi artificiale, che mima la fotosintesi naturale (Fig. 13). Nella fotosintesi artificiale l'acqua viene divisa nei suoi componenti, idrogeno e ossigeno. Purtroppo (o per fortuna, altrimenti avremmo nella Terra atmosfere di idrogeno al posto di oceani di acqua) questa reazione è energeticamente e cineticamente sfavorita. Per osservarla in rese soddisfacenti è necessario utilizzare opportuni catalizzatori e altre componenti moleco- 
lari in quella che rappresenta senza dubbio una delle più affascinanti sfide della chimica moderna.

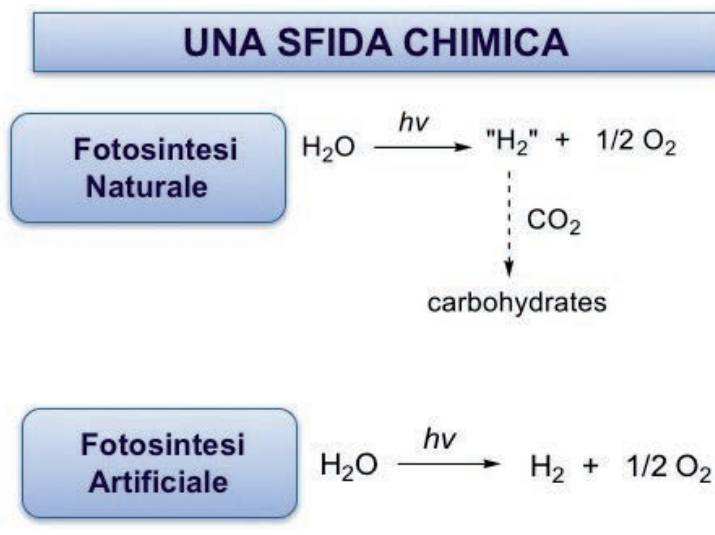

Fig. 13.

Tra i primi catalizzatori studiati, fin dai primi lavori pioneristici degli anni '70,[10] troviamo il biossido di titanio, un materiale economico e largamente utilizzato in molti ambiti commerciali, dalle vernici alle creme solari. Tuttavia il biossido di titanio assorbe solo la porzione ultravioletto dello spettro solare, rimanendo inerte nei confronti della parte dello spettro più importante, quella visibile o colorata (Fig. 14).

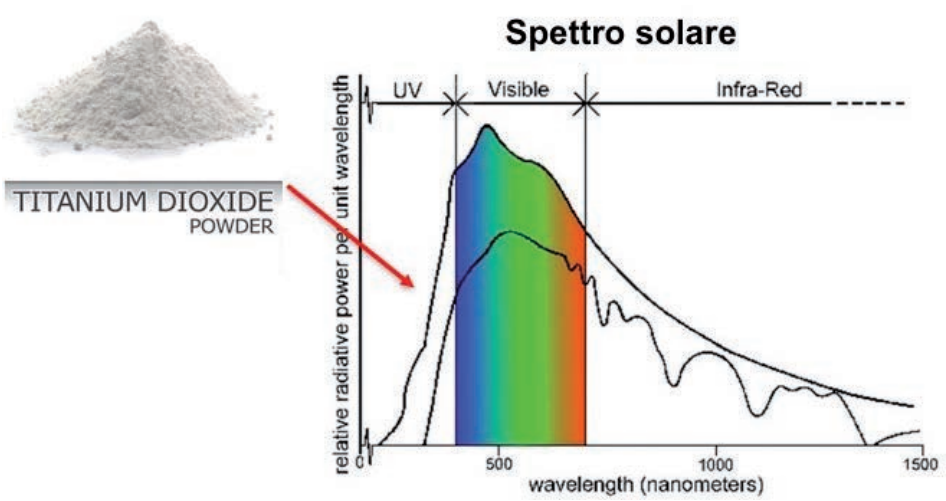

Fig. 14.

E' qui che entrano nuovamente in gioco i coloranti i quali, fungendo da antenne molecolari, catturano efficacemente l'energia solare 
trasferendola ai centri reazionali dove avviene la scissione dell'acqua in idrogeno e ossigeno (Fig. 15).[11] Solo negli ultimi anni sono comparsi in letteratura i primi esempi di coloranti organici per la produzione di idrogeno via fotosintesi artificiale, ricerca che abbiamo recentemente raccolto in una review riepilogativa.[12]

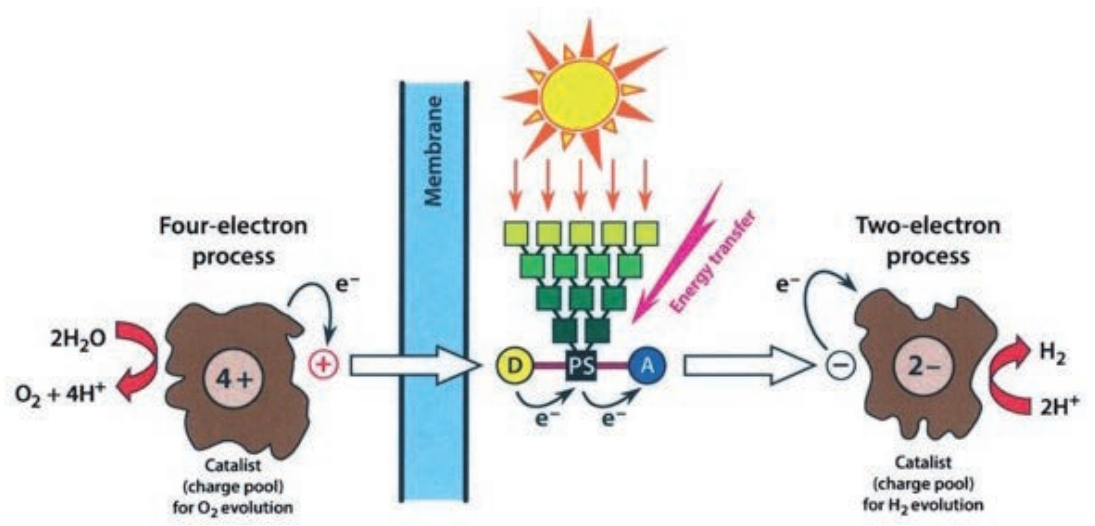

Fig. 15 .

I requisiti progettuali per $\mathrm{i}$ fotosensibilizzatori organici per la produzione di idrogeno sono simili ma non identiche a quelle che abbiamo visto per i coloranti per le applicazioni fotovoltaiche. Con quest'ultimi condividono la necessità di assorbire efficacemente la radiazione solare ma, a differenza di questi, devono lavorare in acqua, un ambiente in genere poco compatibile con le molecole organiche aromatiche, a carattere idrofobo. Inoltre devono possedere legami chimici stabili in tale solvente (ovvero non idrolizzabili) e possibilmente mimare la Natura che ha perfezionato, in milioni di anni, il corrispondente processo naturale della fotosintesi a partire da acqua e sole. Per ottenere questi obiettivi il chimico ingegnerizza nuovamente la struttura molecolare, sia scegliendo opportuni scaffold o templati molecolari, sia sfruttando le funzionalizzazioni periferiche per indurre le proprietà desiderate (Fig. 16).

Le Figg. 17 e 18 riportano alcuni esempi recenti di fotosensibilizzatori sviluppati nei nostri laboratori al MIB-SOLAR.[13] Nel primo caso viene studiato l'effetto della variazione del core elettron-ricco, mentre rimane invariata la rimanente parte della molecola. È interessante osservare che le tre molecole si comportano in maniera differente, 
soprattutto in termini di efficienza energetica e produzione di idrogeno, come conseguenza della, seppur fine, differenza strutturale. Nel secondo esempio vengono invece studiati diversi spaziatori molecolari in corrispondenza dei due rami della molecola multi-ramificata. Anche in questo caso solo certi spaziatori, e non altri, funzionano bene. Nuovamente, solo le molecole col giusto colore presentano le proprietà desiderate. Il risultato è una produzione stabile e continua di idrogeno da acqua e sole, una caratteristica essenziale, come già sottolineato, per lo sviluppo industriale di questi materiali (Fig. 19).

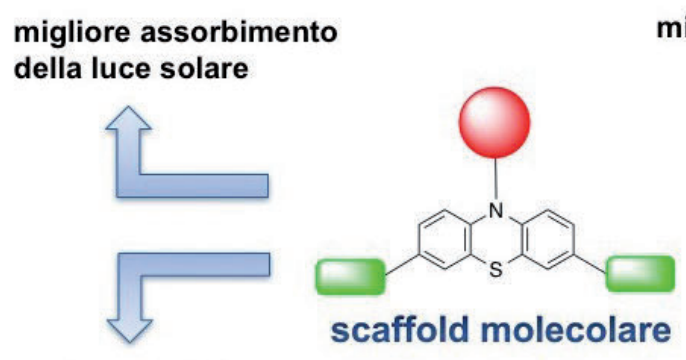

migliore stabilità in ambiente acquoso

migliore bagnabilità

(interfacce)

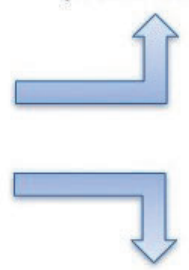

Natura-mimetici

Fig. 16.
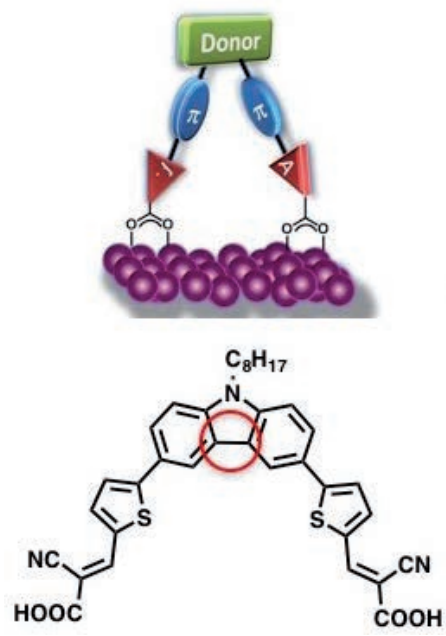
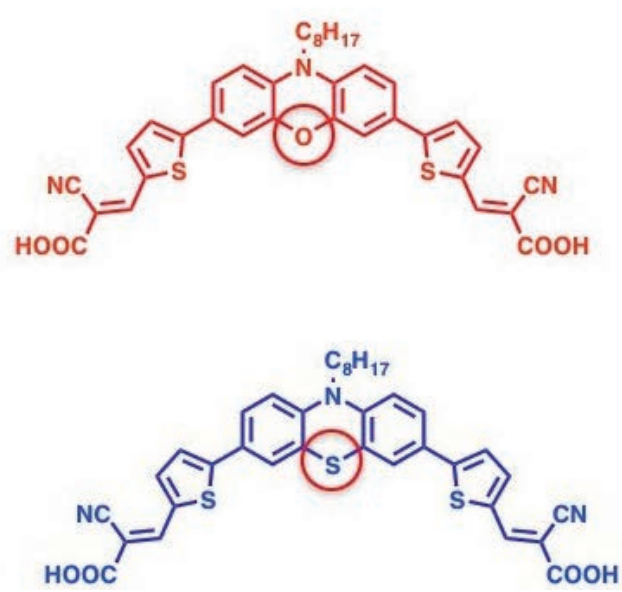

Fig. 17. 


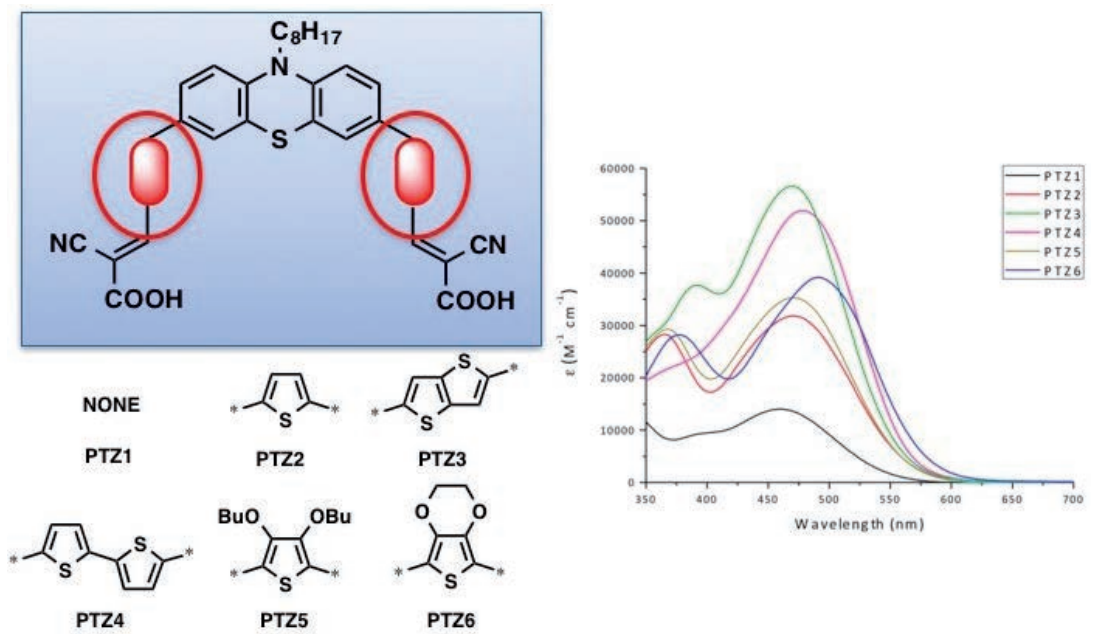

Fig. 18.

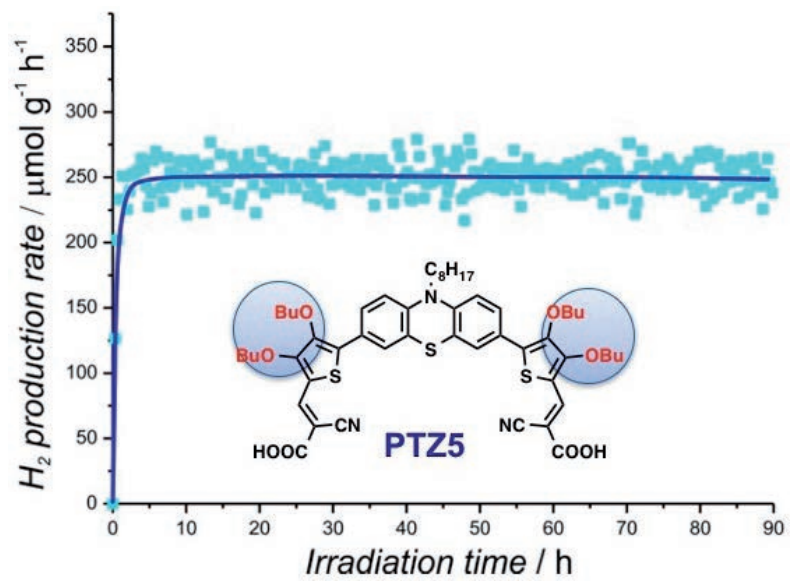

Fig. 19.

Per migliorare l'affinità della molecola all'ambiente acquoso è possibile introdurre in catena laterale delle funzionalità polari idrofile, tali da rendere tutta la molecola idrofila. Anche in questo caso è possibile sfruttare la varietà strutturale a disposizione del chimico, per migliore l'efficienza finale. In un nostro recente lavoro del 2016 (Fig. 20) abbiamo confrontato la capacità di produrre idrogeno utilizzando due coloranti molecolari, entrambi identici tranne che nella porzione periferica idrofi- 
la.[14] Nel primo caso abbiamo utilizzato una catena polare convenzionale (in nero nella Fig. 20), mentre nel secondo caso abbiamo introdotto nella struttura molecolare un pendaglio zuccherino, ed esattamente il frammento del glucosio, che insieme al fruttosio è alla base del saccarosio, o zucchero da cucina. L'idea dietro allo studio è tanto semplice quanto efficace: sfruttare le ben note proprietà idrofile dello zucchero! I risultati confermano quanto sperato: il derivato zuccherino ha una affinità all'acqua ben superiore a quella della semplice catena polare, affinità che ha permesso una produzione di idrogeno praticamente raddoppiata. Gli interessanti risultati hanno valso all'articolo la copertina di una delle riviste chimiche più importanti, forse la più importante, della Royal Society of Chemistry (Fig. 20).

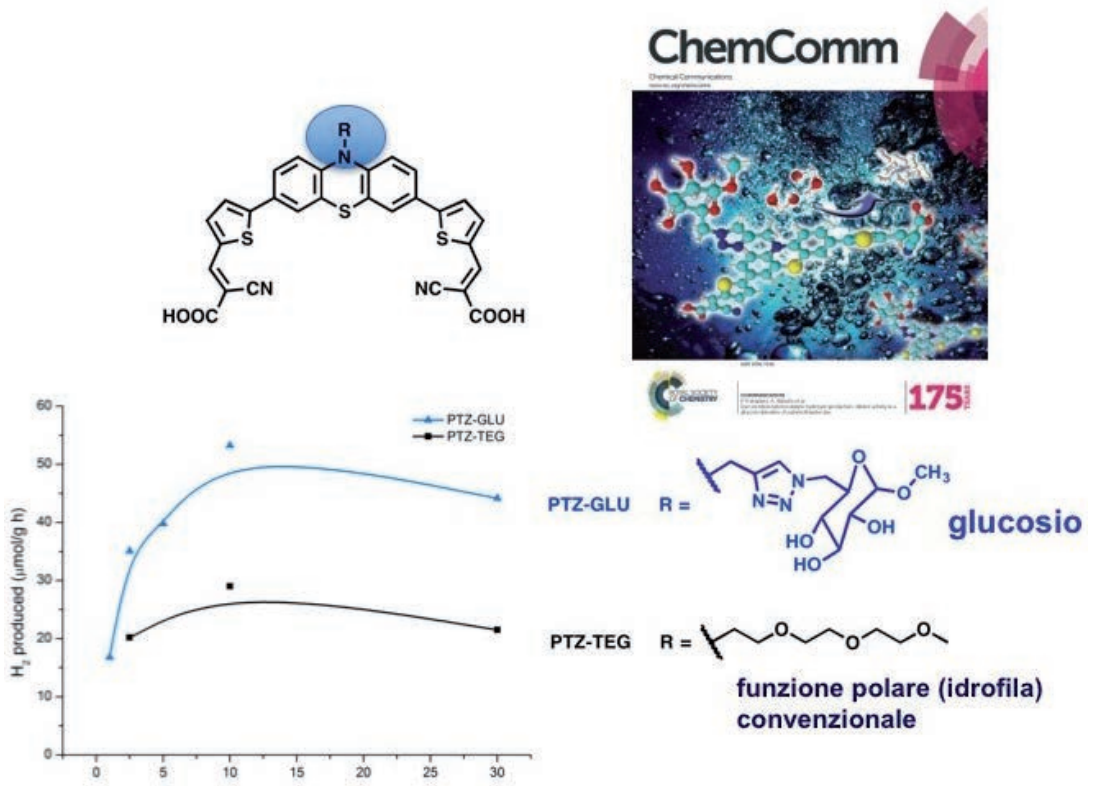

Fig. 20.

Nell'ultimo esempio, descritto in Fig. 21, il target è ancora più ambizioso. Sempre sfruttando il glucosio, ora lo scopo è quello di indurre le molecole a posizionarsi in modo ordinato sulla superficie dell'elettrodo. E' infatti noto che se le molecole sono vicine le une alle altre le prestazioni diminuiscono per un fenomeno di reciproco spegnimento dell'attività (quenching intermolecolare), se le molecole sono ordinate 
parallelamente ma opportunamente spaziate allora le prestazioni sono massimizzate. Non è facile indurre le singole molecole a disporsi nello spazio come noi desideriamo. Nel nostro caso abbiamo sfruttato le deboli interazioni intermolecolari che si stabiliscono spontaneamente tra le molecole dotate del residuo periferico zuccherino e il glucosio stesso (qui aggiunto come acido glucuronico, un suo immediato derivato) (in basso a destra nella Fig. 21).[15] Dopo aver accertato l'ipotesi con sofisticate simulazioni computazionali (in basso a sinistra) abbiamo misurato la produzione di idrogeno senza (colonne rosse) e con (colonne blu) l'aggiunta di acido glucuronico. L'effetto è evidente. Nel secondo caso la produzione di idrogeno è raddoppiata, grazie alla costruzione dell'edificio supermolecolare.

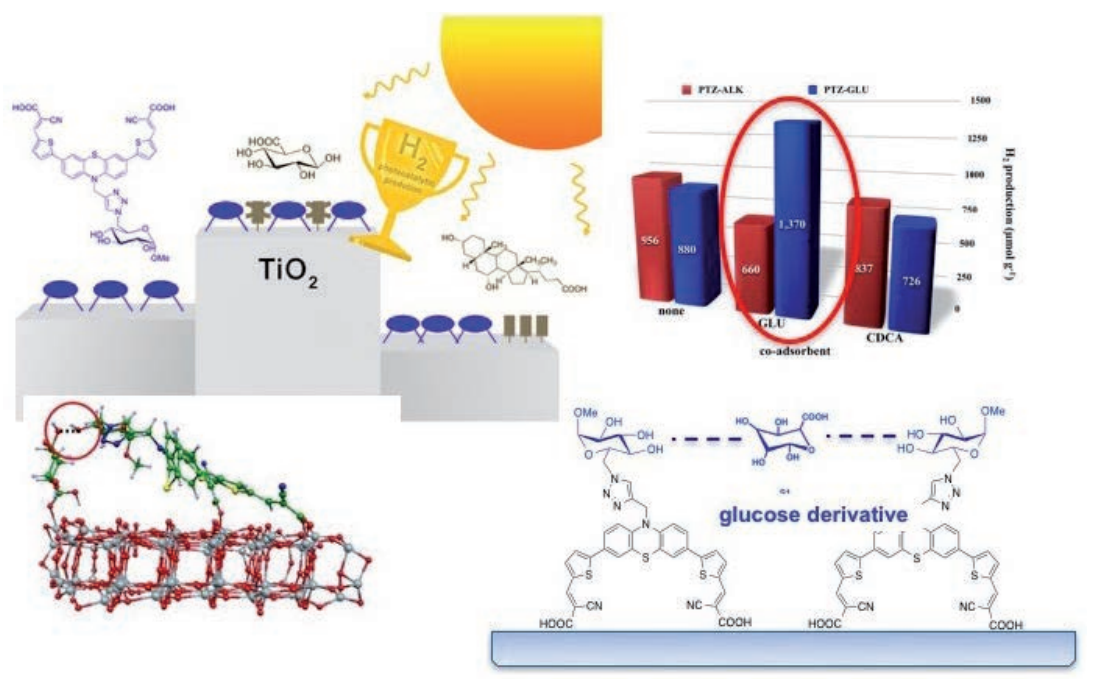

Fig. 21.

\section{CONCLUSIONI}

La chimica molecolare, in particolare la chimica organica, è chiamata a partecipare alle nuove sfide scientifiche e tecnologiche nel campo dell'energia, contribuendo a risolvere le criticità attuali e a definire nuove soluzioni per l'approvvigionamento energetico dei prossimi decenni. Originali molecole innovative vengono oggi progettate per 
rendere i dispositivi solari, sia per produzione di elettricità che per produzione di combustibili, più economici, efficienti, stabili e competitivi con le fonti fossili tradizionali. Pur esistendo in letteratura ormai diversi esempi, questa sfida è solo all'inizio. Da una parte, in termini di prove concettuali, le molecole organiche hanno già dimostrato di poter assumere il ruolo di coprotagonisti nella nuova sfida energetica del nostro secolo, dall'altro ancora molta strada deve essere percorsa, in termini soprattutto di efficienza e stabilità, ad oggi ancora non sufficiente per un impiego di massa ma, come descritto nei precedenti capitoli, in continua evoluzione passo dopo passo. La sfida è certamente complessa e richiederà anni per essere completamente risolta ma, al pari di altre tecnologie a base di molecole organiche, quali ad esempio i cristalli liquidi o gli OLED che hanno richiesto decine di anni prima di arrivare nel mercato, anche in questo campo, alla fine, il ruolo dei coloranti organici sarà senza dubbio rilevante.

\section{RICONOSCIMENTI}

Gli autori desiderano ringraziare l'Università di Milano-Bicocca per il finanziamento che ha permesso la creazione del Centro di Ricerca dell'Energia Solare MIB-SOLAR[16] dove gran parte degli studi descritti sono stati sviluppati (Fig. 22), il Ministero dell'Istruzione, dell'Università e della Ricerca, la Regione Lombardia, l'Unione Europea e ENI SpA per il contributo finanziario alle ricerche qui descritte.

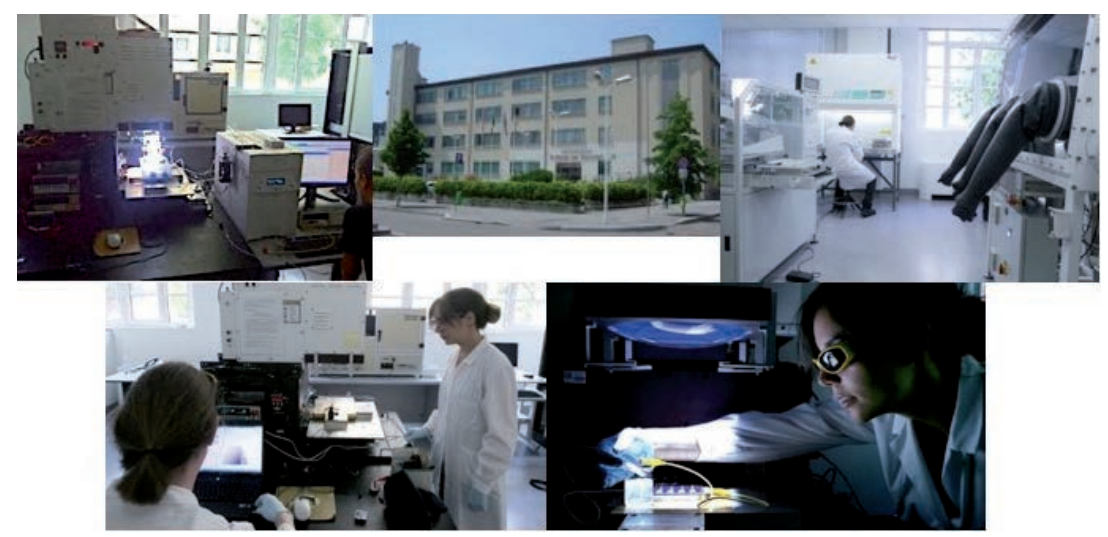

Fig. 22. 


\section{BIBLIOGRAFIA}

1. REN21. 2017. Renewables 2017 Global Status Report (Paris: REN21 Secretariat) (il report è disponibile online sul sito di REN21 (http://www.ren 21.net/).

2. Armaroli N., Balzani V., Energia per l'Astronave Terra, Zanichelli, Bologna, 2017.

3. Fonte: Terna SpA (http://www.terna.it/).

4. Balzani V., Armaroli N., Energy for a Sustainable World: From the Oil Age to a Sun-Powered Future, Wiley-VCH, Weinheim, 2010.

5. https://www.nrel.gov/

6. Manfredi N., Cecconi B., Abbotto A., Multi-Branched Multi-Anchoring MetalFree Dyes for Dye-Sensitized Solar Cells, Eur. J. Org. Chem., 2014: 7069-7086.

7. (a) Abbotto A., Acciarri M., Biagini P., Binetti S., Organic dye for a dye sensitized solar cell. PCT Int. Appl., 2014: WO 2014053626; (b) Biagini P., Abbotto A., Manfredi N., Organic dyes and dye-sensitized solar cells using them, PCT Int. Appl., 2015: WO 2014125432.

8. Gatti T., Manfredi N., Boldrini C., Lamberti F., Abbotto A., Menna E., A D- $\pi$ A organic dye - reduced graphene oxide covalent dyad as a new concept photosensitizer for light harvesting applications, Carbon, 2017: 115: 746-753.

9. Boldrini C. L., Manfredi N., Perna F. M., Trifiletti V., Capriati V., Abbotto A., Dye-Sensitized Solar Cells that use an Aqueous Choline Chloride-Based Deep Eutectic Solvent as Effective Electrolyte Solution, Energy Technology, 2017: 5: 345-353.

10. Fujishima A., Honda K., Electrochemical Photolysis of Water at a Semiconductor Electrode, Nature, 1972: 238: 37-38.

11. (a) Armaroli N., Balzani V., Solar Electricity and Solar Fuels: Status and Perspectives in the Context of the Energy Transition, Chem. Eur. J., 2016: 22: 32-57; (b) Armaroli N., Balzani V., The Hydrogen Issue, Chemsuschem, 2011: 4: 21-36.

12. Cecconi B., Manfredi N., Montini T., Fornasiero P., Abbotto A., Dye-Sensitized Solar Hydrogen Production: The Emerging Role of Metal-Free Organic Sensitizers, Eur. J. Org. Chem., 2016: 5194-5215.

13. Cecconi B., Manfredi N., Ruffo R., Montini T., Romero-Ocaña I., Fornasiero P., Abbotto A., Tuning Thiophene-Based Phenothiazines for Stable Photocatalytic Hydrogen Production, ChemSusChem, 2015: 8: 4216-4228.

14. Manfredi N., Cecconi B., Calabrese V., Minotti A., Peri F., Ruffo R., Monai M., Romero-Ocana I., Montini T., Fornasiero P., Abbotto A., Dye-sensitized photocatalytic hydrogen production: distinct activity in a glucose derivative of a phenothiazine dye, Chem. Commun., 2016: 52: 6977-6980.

15. Manfredi N., Monai M., Montini T., Peri F., De Angelis F., Fornasiero P., Abbotto A., Dye-sensitized Photocatalytic Hydrogen Generation: Efficiency Enhancement by Organic Photosensitizer - Co-Adsorbent Intermolecular Interaction, ACS Energy Letters, 2017; DOI:10.1021/acsenergylett.7b00896.

16. www.mibsolar.mater.unimib.it 